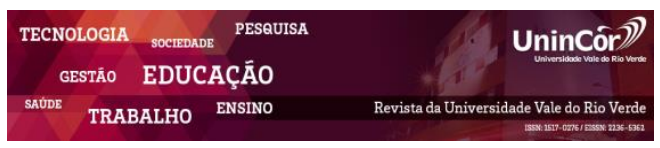

Revista da Universidade Vale do Rio Verde ISSN: 1517-0276 / EISSN: 2236-5362 v. $17 \mid$ n. $1 \mid$ Ano 2019

Aline Santos Moreira

Faculdade Pitágoras de Poços de Caldas aline_sm1@hotmail.com

Bruna Sales

Faculdade Pitágoras de Poços de Caldas bruna_795@hotmail.com

Luciana Belchior Ribeiro

Faculdade Pitágoras de Poços de Caldas lu.belchiorribeiro@gmail.com

Luana Teixeira

Faculdade Pitágoras de Poços de Caldas lu_15teixeira@hotmail.com

Ralph Moraes de Oliveira Faculdade Pitágoras de Poços de Caldas ralph.m@bol.com.br

Maria de Fátima Lino Coelho Faculdade Pitágoras de Poços de Caldas maria.coelho@pitagoras.com.br

Yula de Lima Merola

Faculdade Pitágoras de Poços de Caldas yula.merola@pitagoras.com.br

\section{PESQUISA DE PARASITOS INTESTINAIS \\ EM CRIANÇAS DE UM CENTRO DE EDUCAÇÃ̃O INFANTIL EM UM MUNICÍPIO NO SUL DE MINAS GERAIS}

\section{RESUMO}

As enteroparasitoses são um problema de saúde pública Brasil. Neste contexto os mais afetados por estes parasitos são crianças, por serem um grupo que estão constantemente expostos a locais e atividades que expõe risco de contaminação. No período de agosto a novembro de 2017, analisou-se amostras de fezes de crianças de 0 a 6 anos de um Centro de Educação Infantil em um Município do sul de Minas Gerais. Essas, foram obtidas por doação espontânea após palestra explicativa e assinatura do termo de consentimento pelos pais ou responsáveis, estes também responderam a um questionário para complementar as informações. As amostras foram analisadas pelos métodos de Hoffman, Pons e Janer e Faust e cols. Das 57 amostras analisadas, o índice de positividade foi de $5(8,77 \%)$ e $52(91,23 \%)$ de negatividade, para parasitos intestinais. Dentre as positivas, houve predominância do protozoário Giardia lamblia 3 (60\%). Com o resultado laboratorial em mãos os pais das crianças infectadas foram orientados a procurar um médico inscrito no Sistema Único de Saúde para que fosse possível realizar a clínica da criança e avaliar a necessidade de tratamento. A todos os participantes do projeto foi entregue um panfleto com formas de como fazer a prevenção das parasitoses e normas de higiene de modo a evitar uma futura infecção. Mesmo com um número baixo de parasitos intestinais, reforça-se a necessidade de campanhas educativas sobre as formas de prevenção de parasitoses, sempre com o objetivo de promover a saúde e melhorando a qualidade de vida dessas crianças.

Palavras-Chave: Parasitos intestinais. Exame parasitológico. Crianças. Creches. Diagnóstico laboratorial

\section{RESEARCH OF INTESTINAL PARASITES IN CHILDREN OF A CHILD EDUCATION CENTER IN A MUNICIPALITY IN THE SOUTH OF MINAS GERAIS}


protozoa predominated $(60 \%)$. With the laboratory results in hand, the parents of the infected children were instructed to seek a doctor enrolled in the Unified Health System so that it was possible to perform the child's clinic and evaluate the need for treatment. A pamphlet was given to all project participants with ways to prevent parasitic infections and hygiene standards in order to avoid future infection. Even with a low number of intestinal parasites, there is a need for educational campaigns on ways to prevent parasitic diseases, always with the aim of promoting health and improving the quality of life of these children.

Keywords: Intestinal parasites. Parasitological examination. Children. Creches. Laboratory diagnosis

Recebido em: 19/02/2018 - Aprovado em: 19/03/2019 - Disponibilizado em: 15/07/2019

\section{INTRODUÇÃO}

Parasitoses intestinais são consideradas um grave problema de saúde pública, principalmente em regiões com baixo índice de desenvolvimento econômico e condições e saneamento básico precário (VASCONCELOS et al., 2011). Segundo a Organização Mundial de Saúde (OMS) (2017), doenças parasitárias são responsáveis por um número alarmante de óbitos em todo o mundo.

Geralmente, estas, estão associadas a vários fatores pré-determinantes seja ligados com a sua transmissão, como saneamento básico deficiente, fatores socioculturais, contato com animais, consumo de água e alimentos contaminados com fezes, idade do hospedeiro, entre outros (MANFROI, 2009).

No Brasil, há vários agentes etiológicos responsáveis pelas enteroparasitoses, dentre os helmintos destacam-se os nematelmintos Ascaris lumbricoides e Trichuris trichiura e os ancilostomídeos Necator americanus e Ancylostama duodenale. Dentre os protozoários

Estão Entamoeba histolytica e Giardia duodenalis (COURA, 2005).
Estudos demonstram que um número elevado de casos de enteroparasitoses não são diagnosticados, pois muitas vezes são assintomáticas e a ocorrência dessas infestações leva o indivíduo a várias complicações orgânicas, como deficiência nutricional, baixo desenvolvimento cognitivo e físico, ocasionando risco à saúde (FONSECA, et al., 2010). Entre as doenças mais comuns causadas por parasitos, estão as helmintíases e protozooses, que atingem principalmente crianças em idade pré-escolar.

As crianças em idade escolar são as mais atingidas e prejudicadas pelas doenças parasitárias, uma vez que seus hábitos de higiene são, na maioria das vezes, inadequados e sua imunidade ainda não está totalmente eficiente para a eliminação dos parasitos (MORRONE, CARNEIRO e REIS et al., 2004). As enteroparasitoses colaboram para o agravamento de quadros de desnutrição, diarréia, anemias, diminuição do desenvolvimento físico e do aproveitamento escolar das crianças. No entanto, embora muito se discuta sobre a importância das parasitoses intestinais, principalmente entre crianças em idade escolar, pouca atenção tem sido dada ao assunto nos programas de formação de educadores (PINHEIRO, 2007) 
Portanto, as crianças usuárias das creches estão mais sujeitas ao parasitismo do que aquelas que não as utilizam, e nesta faixa etária o sistema imunológico ainda é muito imaturo, o que as tornam mais susceptível as contaminações (PINHEIRO, 2011).

Nesse contexto, o presente estudo objetivou verificar a importância da realização de estudos sobre a prevalência de parasitos intestinais de uma amostra de crianças de 0 a 6 anos residentes em um município do sul de Minas Gerais.

\section{MATERIAL E MÉTODOS}

A pesquisa foi realizada no Centro de Educação Infantil de um município no sul de Minas Gerais, no período da pesquisa, com 142 crianças de 0 a 6 anos de idade, das quais 57 participaram do trabalho, o que significou uma adesão de $40,14 \%$. A pesquisa foi realizada no período de agosto a novembro de 2017. Inicialmente, realizou-se uma reunião com pais e professores para o esclarecimento do objetivo do trabalho.

Para os pais, foram entregues frascos coletores com instruções de coleta e também o termo de consentimento que, assinado, autorizava as crianças a participarem da pesquisa.

Também aplicou-se para cada participante, um questionário padrão, baseado em um conjunto de questões objetivas e de múltipla escolha, levando-se em consideração a situação de saúde, higiene e saneamento básico.

Para a coleta das amostras de fezes os pesquisadores elaboraram um folheto explicativo, o qual foi distribuído aos pais e/ou responsáveis e cuidadoras, juntamente com os copos coletores.
Após, foram encaminhadas para o laboratório didático de Parasitologia clínica do Curso de Farmácia da Faculdade. Os métodos laboratoriais utilizados para o exame parasitológico de fezes foram: centrífugo-flutuação em solução de sulfato de zinco (Método de Faust) e Sedimentação Espontânea (Método de Lutz, Hoffman ou Pons e Janner). Após a execução do procedimento técnico, as amostras para leitura foram colocadas em lâminas de microscopia, em seguida adicionadas lugol e lamínulas, e em seguida examinadas através da microscopia direta. Para cada amostra foram confeccionadas duas lâminas de cada método, e a leitura foi realizada por dois pesquisadores diferentes.

Os laudos foram entregues aos pais e/ou responsáveis, juntamente com um folder explicativo, sobre as formas de prevenção de parasitoses, pois os parasitos encontrados são marcadores de contaminação fecal-oral. Durante o desenvolvimento do trabalho realizou-se teatro, o qual foi atividade apresentada durante as festividades da semana da criança. A peça foi desenvolvida com o assunto prevenção de parasitoses intestinais. Importante ressaltar que o conteúdo foi adaptado de acordo com a idade das crianças assistidas.

As crianças que apresentaram laudos laboratoriais positivos, os responsáveis foram orientados a procurarem uma Unidade Básica de Saúde (UBS) para a realização de uma avaliação clínica, identificação de sintomatologia e necessidade ou não de tratamento, pois alguns dos protozoários encontrados são considerados não patogênicos.

É um estudo epidemiológico do tipo transversal, onde a detecção da doença foi realizada através do exame parasitológico de 
fezes. A exposição ao risco foi determinada pela aplicação de questionários padrão. Os dados são apresentados em percentagens referentes à prevalência da doença e/ou fatores predisponentes ao desenvolvimento de enteroparasitoses. Foi realizada uma análise descritiva dos fatores de risco as parasitoses intestinais. As amostras de fezes e dados de exposição ao risco foram coletadas simultaneamente.

Ao final da pesquisa os dados foram digitados e tabulados em um banco de dados para a execução da análise dos mesmos. O banco de dados, bem como as tabelas e gráficos foram construídos no Microsoft Excel® 2010.

$\mathrm{O}$ presente projeto foi autorizado pelo Comitê de Ética (CAEE: 60312016.0.0000.5102), e teve prévia autorização dos pais ou responsáveis pelos escolares envolvidos.
Das 57 crianças que participaram do estudo, a idade média era de 3 anos e 9 meses, das quais $33(57,9 \%)$ eram do gênero feminino, sendo que $3(60 \%)$ apresentaram resultados positivos de infecção por protozoários e $24(42,1 \% \%)$ crianças do gênero masculino, 2 (40\%) estavam infectadas também por protozoários. A prevalência total foi de $5(8,8 \%)$ crianças com resultado positivo para parasitos intestinais. Não foram encontradas diferenças significativas para as variáveis idade e gênero.

Dentre os parasitos intestinais encontrados todos foram protozoários. Das 5 crianças parasitados, 3 (60\%), encontravam-se parasitados pelo protozoário Giardia lamblia, 1 (20\%), Entamoeba coli e 1 (20\%) Endolimax nana. Não havendo presença de bioparasitismo ou poliparasitismo, conforme tabela 1.

\section{RESULTADOS E DISCUSSÃO}

Tabela 1 - Frequência dos parasitos intestinais de acordo com o gênero das crianças.

\begin{tabular}{|c|c|c|c|}
\hline POSITIVIDADE & $\begin{array}{c}\text { MASCULINO } \\
\text { n(\%) }\end{array}$ & $\begin{array}{c}\text { FEMININO } \\
n(\%)\end{array}$ & $\begin{array}{l}\text { TOTAL } \\
\text { n(\%) }\end{array}$ \\
\hline Giardia lamblia & $2(40 \%)$ & $1(20 \%)$ & $3(60 \%)$ \\
\hline Entamoeba coli & - & $1(20 \%)$ & $1(20 \%)$ \\
\hline Endolimax nana & $1(20 \%)$ & - & $1(20 \%)$ \\
\hline
\end{tabular}

FONTE: Os autores.

De acordo com os parasitos encontrados (Tabela 1), observou-se maior frequência de cistos de Giardia lamblia (60\%), prevalecendo sobre o gênero masculino. Não foram encontrados casos de poliparasitismo entre as amostras analisadas.

Os protozoários Entamoeba coli e Endolimax nana, são considerados parasitos não patogênicos, entretanto apresentam grande importância no exame parasitológico de fezes. Estes, indicam que o ambiente está com contaminações fecais, e as crianças estão expostas, bem como é possível que haja contaminação da água e de alimentos (BORGES, 2011).

Em relação ao parasito encontrado Giardia lamblia, este é um parasito cujos cistos do protozoário são resistentes ao tratamento da água cloro (BORGES et al., 2011) e a transmissão é 
comum entre as crianças, devido esses cistos serem infectantes quando eliminados nas fezes (SOGAYAR \& GUIMARÃES, 2005), e presente no solo e água são de fácil contaminação.

Em relação à positividade encontrada, quanto se observou em relação ao gênero encontrou-se $3(60 \%)$ do gênero masculino e 2 (40\%) sendo do gênero feminino. Kamagone, et al.,(2007), constatou em seu estudo que o gênero feminino seria um fator de proteção pois tem 0,2 vezes menos chance de se infectar com alguma parasitose já que crianças do gênero masculino ficam mais expostas ao ambiente circundante de suas casas em suas atividades de lazer tendo mais contato com o solo onde associado à menor higiene aumentam as chances de infecção.

No presente estudo, identificou-se que na análise parasitológica, as crianças apresentaram uma baixa positividade $8,8 \%$, para parasitos intestinais, sendo o protozoário Giardia lamblia, o parasito de maior frequência 60\%. Dados semelhantes foram encontrados em trabalhos realizados por Magalhães, et al., (2013), 24,7\%; Zaiden, et al.,(2008), 21,4\% e de 68,8\% descrito por Lopes, Salamaia e Molinari (2012). Seguido por Entamoeba coli 1 (20\%) e Endolimax nana 1 (20\%).

Já Santos (2014), em um estudo semelhante realizado em 2014, no município de Florianópolis - SC, encontrou um número elevado de amostra positivas $35(61,4 \%)$, entretanto, notase diferenças de condições sanitárias entre os dois municípios, motivo que favorece um baixo índice de positividade nas amostras do presente estudo.
Pode-se observar que nos trabalhos de Rocha, et al., (2000), Batista, et al., (2009), Komagome, et al., (2007), Chaves, et al., (2006) e Ramos (2006), em que as porcentagens dos valores negativos foram, respectivamente, $78.4 \%, 70.7 \%$, $65.5 \%, 61.6 \%$ e 58\%. Quando comparados os dados observa-se que os resultados obtidos nesta pesquisa também se mostram com valores de $91,2 \%$ de negatividade, refletindo um dado importante de saúde pública.

Por outro lado os valores se aproximam relativamente de um estudo realizado por Bevilacqua, et. al., (2009) em Taubaté - SP, 15\% $(n=18)$ de positividade em análise de uma creche localizada naquele município com faixa etária entre 0 a 6 anos. Dados semelhantes de positividade, foram encontrados por Fernandez (2006), realizado na cidade de Poços de Caldas Minas Gerais com um índice de $12,5 \%$.

A análise parasitológica realizada em crianças de 0 a 6 anos que frequentam creches são de extrema importância, pois além de serem observadas as condições de saneamento básico da região, é nesta faixa etária que existe uma maior probabilidade da ocorrência de parasitoses devido à falta de compreensão sobre hábitos de higiene pessoal (ZAIDEN, et al., 2008).

De acordo com a tabela 2, são descritas as variáveis relacionadas às condições socioeconômica e de higiene das famílias atendidas na presente pesquisa. 
Tabela 2- Dados relacionados aos hábitos de higiene e perfil socioeconômico.

\begin{tabular}{|c|c|c|}
\hline$\overline{\text { VARIÁVEIS }}$ & $\mathbf{N}$ & $\%$ \\
\hline \multicolumn{3}{|l|}{ IDADE ESCOLAR } \\
\hline Berçário (0 a 1 ano) & 9 & $16 \%$ \\
\hline Maternal II (1 a 2 anos) & 3 & $5 \%$ \\
\hline Maternal III ( 2 a 3 anos) & 22 & $39 \%$ \\
\hline Jardim I (3 a 4 anos) & 13 & $23 \%$ \\
\hline Jardim II (4 a 6 anos) & 10 & $17 \%$ \\
\hline \multicolumn{3}{|c|}{ RENDA FAMILIAR (salário mínimo) } \\
\hline 1 & 5 & $9 \%$ \\
\hline $1^{1 / 12}$ & 3 & $5 \%$ \\
\hline 2 & 7 & $12 \%$ \\
\hline 3 & 17 & $30 \%$ \\
\hline 4 & 10 & $18 \%$ \\
\hline Outros & 8 & $14 \%$ \\
\hline Não respondeu & 7 & $12 \%$ \\
\hline \multicolumn{3}{|l|}{ REGIÃO DE MORADIA } \\
\hline Urbana & 57 & $100 \%$ \\
\hline \multicolumn{3}{|l|}{ ÁGUA ENCANADA } \\
\hline Sim & 56 & $98 \%$ \\
\hline Não & 1 & $2 \%$ \\
\hline \multicolumn{3}{|c|}{ FRUTAS E LEGUMES SEMPRE SÃO SEMPRE HIGIENIZADOS ANTES DA PREPARAÇÃO } \\
\hline Sim & 56 & $98 \%$ \\
\hline Não & 1 & $2 \%$ \\
\hline \multicolumn{3}{|c|}{ A ÁGUA É FILTRADA OU FERVIDA ANTES DO CONSUMO } \\
\hline Sim & 52 & $91 \%$ \\
\hline Não & 5 & $9 \%$ \\
\hline \multicolumn{3}{|c|}{ RECOLHIMENTO DE LIXO FREQUENTE } \\
\hline Sim & 57 & $100 \%$ \\
\hline \multicolumn{3}{|c|}{ SANEAMENTO BÁSICO } \\
\hline Sim & 56 & $98 \%$ \\
\hline Não & 1 & $2 \%$ \\
\hline \multicolumn{3}{|l|}{ POSSUI BANHEIRO } \\
\hline Sim & 57 & $100 \%$ \\
\hline \multicolumn{3}{|c|}{ TEM ANIMAIS DE ESTIMAÇÃO } \\
\hline Sim & 26 & $46 \%$ \\
\hline Não & 31 & $54 \%$ \\
\hline \multicolumn{3}{|c|}{ FEZ EXAME PARASITOLÓGICO NOS ÚLTIMOS MESES } \\
\hline Sim & 15 & $26 \%$ \\
\hline Não & 42 & $74 \%$ \\
\hline
\end{tabular}

FONTE: Os autores.

Dos 57 participantes que responderam ao questionário, em relação à renda familiar, esta variou de $5 \%$ a $30 \%$, onde observou-se que a maioria dos entrevistados recebia em torno de 3 salários mínimos.

Já Figueiredo e Querol (2011), em relação à renda familiar $16(53,3 \%)$, possuíam renda familiar de um salário mínimo. Enquanto na atual pesquisa somente $5(9 \%)$ possuíam essa renda.
Dos entrevistados $56(98 \%)$ responderam que faziam uso de água encanada, 57 (100\%) e tinham acesso ao lixo urbano e $52(91 \%)$ faziam uso de água filtrada. Dados semelhantes foram encontrados por Rech, et al., (2016), onde , 94,2\% consomem água potável, 64,7\% possuem coleta e tratamento de esgoto e 94,2\% afirmaram ter coleta de lixo.

Segundo Carvalho Gomes (2013), em sua pesquisa verificou que $70 \%$ dos pesquisados lavavam as frutas e verduras apenas com água. 
Dados um pouco mais altos, foram verificados na atual pesquisa, onde 56 (98\%) dos entrevistados disseram que higienizam frutas e verduras antes do preparo, assim como concluiu-se que $91 \%$ das crianças fazem uso de água fervida ou filtrada antes do consumo.

De acordo com a presença de animais de estimação na residência, a presente pesquisa identificou que 26 (46\%) responderam que tinham e 31 (54\%) não. Já Carvalho e Gomes (2011), encontraram em sua pesquisa que $77 \%$

dos escolares tinham contato constante com animais domésticos. Reuter, et al., (2015), encontrou 74,2\%, entretanto Zaiden (2008), $25,7 \%$. Importante ressaltar que na presente pesquisa a pergunta foi direcionada para saber se havia a presença de animais domésticos em casa (cães).

Em relação a realizarem análises de fezes nos últimos meses, 15 (26\%), responderam que fizeram a análise, enquanto 42 (74\%), não haviam realizado o exame. Destes, $24(42,1 \%)$ eram do gênero masculino e $33(57,9 \%)$ do gênero feminino. 5 (8,8\%) de positividade e $52(91,2 \%)$ de negatividade á presença de parasitos intestinais.

Apesar de isoladamente não apresentarem alta letalidade, as enteroparasitoses podem ser consideradas cofatores da mortalidade infantil, considerando que infecções por parasitos intestinais podem afetar o equilíbrio nutricional, induzir sangramento intestinal e má absorção de nutrientes, além de competir pela absorção de micronutrientes, reduzir a ingesta alimentar (MELO et al., 2010).

Entretanto, nesta pesquisa, o baixo índice de positividade para parasitos intestinais, pode ser justificado, pelo fato do município em estudo, ser contemplado com um alto nível de cobertura de saneamento básico, pois o município possui um órgão próprio para tratamento de água, e segundo o órgão, em 2010, a cidade já contava com várias estações de tratamento todas dotadas de um sistema de fluoretação. Ainda para o órgão no ano de 2010, 100\% das residências já possuíam acesso à água tratada e 99,2\% ligação de esgoto.

\section{CONCLUSÃO}

Quanto às condições de moradia, observouse que a maioria apresentava condições adequadas, faziam uso de água encanada, tomavam água filtrada, lavavam as frutas antes de ingeri-las e afirmaram também que tinham coleta de lixo.

Diante dos resultados obtidos no Centro de Educação Infantil no município em estudo, a frequência de crianças com parasitoses intestinais foi baixa. Sendo a Giardia lamblia o protozoário de maior prevalência. Importante ressaltar que está associado a complicações diversas, como quadros de diarréia crônica e desnutrição.

Os resultados obtidos neste trabalho foram satisfatórios, apresentando um baixo índice positivo. A escolha dos métodos laboratoriais sensíveis, específicos e de fácil preparação é fundamental para o diagnóstico individual. Porém, de acordo com De Carli (2001), é permissível que haja alguma influência nos resultados devido ao ciclo reprodutivo dos parasitos, pois exames de amostras múltiplas aumentam a possibilidade de resultados positivos devido aos vários fatores como, por exemplo, os diferentes estágios dos protozoários, visto que no presente trabalho foi coletada apenas uma amostra de cada indivíduo. 
Mesmo com um número baixo de parasitos intestinais, reforça-se a necessidade de campanhas educativas sobre as formas de prevenção de parasitoses, sempre com o objetivo de promover a saúde e melhorar qualidade de vida dessas crianças.

\section{AGRADECIMENTOS}

Aos pais e/ou responsáveis, professores e coordenação do Centro de Educação Infantil pela compreensão do projeto realizado.

\section{REFERÊNCIAS}

BATISTA, Thaís et al. Parasitoses intestinais em préescolares matriculados em creche filantrópica no sul de Santa Catarina. Arquivos Catarinenses de Medicina Vol. 38, nº 3, 2009.

BEVILACQUA, Ana Amélia et al. Prevalência de enteroparasitas em crianças de 0 a 6 anos de idade de uma creche do município de Taubaté - SP. Acesso em: 29 jan. 2017 Disponível em: http://www.inicepg.uni-

vap.br/cd/INIC_2009/anais/arquivos/0120_1086_02.p $\underline{\mathrm{df}}$

\section{BORGES WF, MARCIANO FM, OLIVEIRA HB.} Parasitos intestinais: elevada prevalência de Giardia lamblia em pacientes atendidos pelo serviço público de saúde da região sudeste de Goiás, Brasil. Rev Patol Trop 40: 149-157, 2011.

CARVALHO, S. D. E. N.; GOMES, P. N. Prevalência de enteroparasitoses em crianças na faixa etária de 6 a 12 anos na escola pública Melvin Jones em TeresinaPI Revista. Interdisciplinar. v.6, n.4, p.95-101, out.nov.dez. 2013

CHAVES, E. M. S. et al. Levantamento de Protozoonoses e Verminoses nas sete creches municipais de Uruguaiana, Rio Grande do Sul - Brasil. RBAC, vol. 38(1): 39-41, 2006.

COURA, J. R. Dinâmica das doenças parasitárias. Rio de janeiro: Guanabara Koogan, v. 22005.

De Carli GA. Parasitologia Clínica: Seleção de técnicas e métodos de laboratório para o diagnóstico de parasitoses humanas. Atheneu. São Paulo, 2011

FERNADEZ, Silvia Cristina Lopes - "Avaliação epidemiológica de parasitoses intestinais entre escolares assistidos por micro-áreas de unidades de saúde do município de Poços de Caldas - MG” 2006

FIGUEIREDO, M. I. O.; QUEROL, H. Levantamento das parasitoses intestinais em crianças de 4 a 12 anos e funcionários que manipulam o alimento de um centro socioeducativo de Uruguaiana, RS, Brasil. Biodiversidade Pampeana: PUCRS, v. 9, n. 1, p. 3-11, dez., 2011, ISSN 1679-6179.

FONSECA, E.O.L, et al. Prevalência e fatores associados às geo-helmintíases em crianças residentes em municípios com baixo IDH no Norte e Nordeste brasileiros (2010).

KOMAGOME, S. H. et al. Fatores de risco para infecção parasitária intestinal em crianças e funcionários de creche. CiencCuidSaude2007;6 (Suplem. 2): 442447. 2007.

LOPES, CR; SALAMAIA, FH; MOLINARI-CAPEL, LM. Diferentes Parasitos Intestinais em Crianças de Um a Dez Anos Atendidas em um Laboratório de Análises Clínicas na Cidade de Marialva, Paraná, Brasil. Saúde e Pesquisa, 2012

MANFROI A, Stein AT, Castro Filho ED, Abordagem das Parasitoses Intestinais mais Prevalentes na Infância,Projeto Diretrizes Associação Médica Brasileira e Conselho Federal de Medicina, 2009.

MELO, Erenilson Moreira; FERRAZ, Fabiana Nabarro; ALEIXO, Denise Lessa. Importância do estudo da Prevalência de parasitos Intestinais de crianças em idade escolar. SaBios-Revista de Saúde e Biologia, (S.1.), v. 5, n. 1, ago. 2010. ISSN 1980-0002. Disponível em:

HTTP://revista.grupointegrado.br/revista/index.php/sa bios2/article/view/546>. Acesso em:13fev.2018

MORRONE, F.B.; CARNEIRO, J. A.; REIS, C.; et al. Study of enteroparasites infection frequency and chemotherapeutic agents used in pediatric patients in a community living in Porto Alegre, RS, Brazil. Revista do Instituto de Medicina Tropical de São Paulo, São Paulo, v. 46, n. 2, p. 77- 80, jan./fev. 2004.

OMS, Organização Mundial da Saúde. Relatório da OMS informa progressos sem precedentes contra doenças tropicais negligenciadas. Acesso em: $01 \mathrm{de}$ maio. Disponível em: http://www.paho.org/bra/index.php?option $=$ com content $\&$ view $=$ article\&id=5401:relatorio-da-oms-informa-progressossem-precedentes-contra-doencas-tropicais-gligenciadas\&catid=1272: noticiasdtent $\&$ Itemid=816

PINHEIRO, Patrícia Lopes. Enteroparasitoses na infância, seus determinantes sociais e principais conseqüências: uma revisão bibliográfica. Universidade $\mathrm{Fe}$ deral de Minas Gerais, Faculdade de Medicina. Núcleo de Educação em Saúde Coletiva. Governador Valadares, 2011. 
PINHEIRO, R. O. Ocorrência de parasitas entre crianças do pré-escolar de duas escolas em Vassouras, RJ. Revista Brasileira de Farmacia, Rio de Janeiro, v. 88, n. 2, p. 98-9, abr./jun. 2007.

RAMOS, Gloria Cristina Scovino De Castro. Correlação entre parasitoses intestinais, estado nutricional, condições socioeconômicas e sanitárias de crianças de três creches públicas do município de Niterói. Dissertação de Mestrado (Mestrado em Patologia - Área de Concentração: Patologia Clínica e Análises Clínicas Curso de Pós-Graduação em Patologia - Universidade Federal Fluminense), 2006.

RECH, S.C et al. Frequência de enteroparasitoses e condições socioeconômicos de escolares da cidade de São Marcos-RS. Rev Semina: Ciências Biológicas e da Saúde, Londrina, v.37, n.1, p. 25-32, jan/jun. 2016.

REUTER, Cézane Priscila; FURTADO, Lúcia Beatriz Fernandes da Silva; DA SILVA, RAFAELA; PASA, Luiza KLINGER, Elisa Inês; DOS SANTOS, CLAIRTON EDINEI; RENNER, Jane Dagmar Pollo. Frequência de Parasitoses Intestinais: um estudo com crianças de uma creche de Santa Cruz do Sul - RS. Cinergis, v.16,p.142-147,2015.

ROCHA, R. S. et al. Avaliação da esquistossomose e de outras parasitoses intestinais, em escolares do município de Bambuí, Minas Gerais, Brasil. Revista da Sociedade Brasileira de Medicina Tropical 33(5):431436, set-out, 2000.

SANTOS, Juliano dos et al.,. Parasitoses Intestinais em crianças de creche comunitária em Florianópolis, SC, Brasil. Revista de Patologia Tropical, v.43, n.3, p.332-340, out.2014. ISSN 1980 -8178. Disponível em :

HTTPS://www.revsitas.ufg.br/iptsp/article/view/3220 1/17234> Acesso em: 13 fev.2018.

SOGAYAR MITL, GUIMARÃES S. Giardia. In: Neves DP, Melo AL, Genaro O, Linardi PM. Parasitologia humana. Atheneu. Rio de Janeiro, 2005

VASCONCELOS IAB, OLIVEIRA JW, CABRAL FRF, COUTINHO HDM, MENEZES IRA. Prevalência de parasitoses intestinais entre crianças de 4-12 anos no Crato, Estado do Ceará: um problema recorrente de saúde pública. Acta Sci Health Sci.2011.

ZAIDEN, M. F. et al. Epidemiologia das parasitoses intestinais em crianças de creches de Rio Verde - GO. Medicina (Ribeirão Preto) 2008; 41 (2): 182-7.

SILVA, DCB. Avaliação da ventilação mecânica utilizada em unidade de terapia intensiva pediátrica e os fatores de risco: em busca de uma melhor prática ventilatória [Dissertação de Mestrado]. São Paulo: Universidade de São Paulo, 2009 [acesso em 15 maio 2011]. Disponível em: http://www.teses.usp.br.com.br
VIANA, FP. et al. Aspectos epidemiológicos das crianças com queimaduras internadas no Pronto Socorro para queimaduras de Goiânia - Goiás. Rev. Eletr. Enf. [Internet], 2009 [acesso em 14 maio 2011]; 11 (4): 779-784. Disponível em: < http://www.fen.ufg.br/revista/v11/n4/pdf/v11n4a02.pd f>.

\begin{tabular}{l}
\hline Aline Santos Moreira \\
Discente do curso de farmácia da Faculdade \\
Pitágoras - Poços de Caldas (MG) \\
\hline
\end{tabular}

Bruna Sales
Discente do curso de farmácia da Faculdade
Pitágoras - Poços de Caldas (MG)

\section{Luciana Belchior Ribeiro}

Discente do curso de farmácia da Faculdade Pitágoras - Poços de Caldas (MG)

Luana Teixeira
Discente do curso de farmácia da Faculdade
Pitágoras - Poços de Caldas (MG)

\section{Ralph Moraes de Oliveira}

Discente do curso de farmácia da Faculdade Pitágoras - Poços de Caldas (MG)

\section{Maria de Fátima Lino Coelho \\ Docente do curso de farmácia da Faculdade Pitágoras - Poços de Caldas (MG)}

\author{
Yula de Lima Merola \\ Docente do curso de farmácia da Faculdade \\ Pitágoras - Poços de Caldas (MG)
}

